\title{
SHIELDING AGAINST THE NEUTRONS PRODUCED WHEN 400-MeV ELECTRONS ARE INCIDENT ON A THICK COPPER TARGET $\dagger$
}

\author{
R. G. ALSMILLER, JR. and J. BARISH \\ Oak Ridge National Laboratory, Oak Ridge, Tennessee 37830, USA
}

\begin{abstract}
Neutron-transport calculations have been carried out using the method of discrete ordinates, and the dose equivalent as a function of radius in spherical shields of various compositions is presented. The incident-neutron spectra used are characteristic of the spectra emitted in the angular intervals of 0 to $30^{\circ}, 30$ to $60^{\circ}, 60$ to $120^{\circ}$, and 120 to $180^{\circ}$ when $400-\mathrm{MeV}$ electrons are incident on a thick copper target. Results are given for shields of soil, concrete, ilmenite, iron, and iron followed by soil.
\end{abstract}

\section{INTRODUCTION}

When a high-energy electron beam enters a thick target, an electron-photon cascade is initiated and the photons of the cascade interact with the nuclei of the target to produce photoneutrons. It is primarily the higher energy photoneutrons that must be shielded against. An estimate of the energy and angular distribution of the photoneutrons that are produced when high-energy electrons are incident on a thick copper target has been given by Gabriel and Alsmiller ${ }^{1}$ and by Gabriel. $^{2}$ In the present paper, the results of neutron-transport calculations obtained using as a source the neutron spectra produced when 400$\mathrm{MeV}$ electrons are incident on a thick copper target ${ }^{2}$ are presented.t The calculations were carried out using the method of discrete ordinates, and the results obtained are similar to those presented previously for a variety of source spectra..$^{3-5}$ Only shields of soil and concrete, however, were treated previously, while here shields of soil, concrete, ilmenite, iron, and iron followed by soil are considered. Also, the geometry used herea point isotropic source at the center of a spherical shield-is different from the slab geometry that was used previously. ${ }^{3-5}$

In Sec. 2 the calculational details are given. In Sec. 3 the results are presented and discussed.

$\dagger$ This research was funded by the U.S. Atomic Energy Commission under contract with the Union Carbide Corporation.

$\ddagger$ The results presented here were obtained to aid in the design of the shielding for the $400-\mathrm{MeV}$ electron accelerator under construction at the Laboratory of Nuclear Science of the Massachusetts Institute of Technology.

\section{CALCULATIONAL DETAILS}

All of the results presented here were obtained with the one-dimensional discrete ordinates code ANISN. ${ }^{6}$ The geometry considered is that of a point isotropic source at the center of a sphere. The spectra of neutrons emitted when a $400-\mathrm{MeV}$ electron beam is incident on a thick copper target were taken from Ref. 2 and are shown in Fig. 1

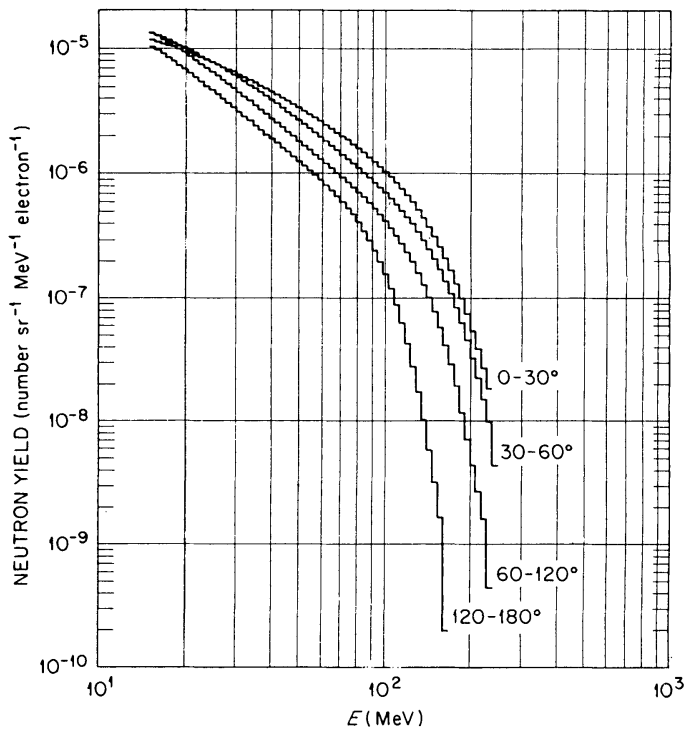

FIG. 1. Neutron yield, averaged over the indicated angular intervals, when $400-\mathrm{MeV}$ electrons are incident on a thick copper target.

for neutron energies $>15 \mathrm{MeV}$. The dimensions of a 'thick' copper target are small compared to the shield thicknesses of interest here, and thus the copper target may reasonably be approximated to be a point. There are, of course, neutrons emitted from the target at energies $<15 \mathrm{MeV}$, but the 
number and energy distribution of these neutrons are not available and are not taken into account. This means that consideration must be restricted to sufficiently thick shields that these low-energy neutrons from the target may be assumed to make no contribution. It must be emphasized that only the low-energy neutrons from the copper target are neglected; production and transport of low-energy neutrons $(<15 \mathrm{MeV})$ in the shield are treated in detail. The angular intervals over which the spectra have been averaged are indicated in the figure. The angles are measured with respect to the direction of motion of the incident electrons. The angular distribution of the neutrons emitted from the copper target is not isotropic, and therefore the problem of transporting these neutrons through a spherical shield is not a one-dimensional problem. Here the approximation is made that each of the spectra shown in Fig. 1 may be treated independently; i.e., a separate calculation is made when each of the spectra shown are isotropically emitted from a point at the center of a spherical shield.

Calculations have been carried out for shields of soil, concrete, ilmenite, iron, and iron followed by soil. Soil was assumed to be composed of $\mathrm{SiO}_{2}$ with 5 per cent $\mathrm{H}_{2} \mathrm{O}$ by weight. The composition of the concrete used in the calculations is the same as that used previously, ${ }^{5}$ and ilmenite was assumed to be $\mathrm{FeTiO}_{3}$. The densities of the shield materials are shown in Table $\mathrm{I}$.

TABLE I

Densities of shield materials

\begin{tabular}{lc}
\hline Shield material & $\begin{array}{c}\text { Density } \\
\left(\mathrm{g} \mathrm{cm}^{-3}\right)\end{array}$ \\
\hline Soil & 1.8 \\
Concrete & 2.3 \\
Ilmenite & 3.8 \\
Iron & 7.8 \\
\hline
\end{tabular}

For neutron energies $<15 \mathrm{MeV}$, the differential elastic and nonelastic cross-section data for $\mathrm{H}, \mathrm{C}$, $\mathrm{N}, \mathrm{O}, \mathrm{Mg}, \mathrm{Al}, \mathrm{Si}$, and $\mathrm{Ca}$ were taken from the RSIC Data Library Collection ${ }^{7}$; for Ti, from the work of Pennington and Gajniak ${ }^{8}$; and for $\mathrm{Fe}$, from ENDF/B MAT. 4180 MOD. $1 . \dagger$ For neutron energies $>15 \mathrm{MeV}$, differential nonelastic crosssection data based on the work of Bertini were

$\uparrow$ Available from the Radiation Shielding Information Center (RSIC) of the Oak Ridge National Laboratory. used. $^{9-11}$ For neutron energies $>15 \mathrm{MeV}$, neutron-hydrogen elastic differential cross-section data were taken from the analytic fits to experimental data given by Bertini. ${ }^{12}$ For neutron energies $>15 \mathrm{MeV}$, the differential elastic crosssection data for collisions with elements other than hydrogen and titanium were taken from the $\mathrm{O} 5 \mathrm{R}^{13}$ master cross-section tape. $\$$ In the case of titanium, differential elastic-scattering cross-section data for neutron energies $>15 \mathrm{MeV}$ were not available, and it was assumed that this elastic scattering could be neglected. In all of the calculations reported here, the protons produced by neutron-nucleus collisions have not been transported since it has previously been shown that the neutrons produced by these protons may, to a good approximation, be neglected. ${ }^{3}$

The dose equivalent as a function of depth in the shield was obtained using the calculated omnidirectional neutron fluence per unit energy and the neutron fluence-to-dose-equivalent conversion factor recommended by the International Commission on Radiation Units and Measurements. ${ }^{14}$

\section{RESULTS AND DISCUSSIONS}

In presenting the results, the radial distance into the shield is often measured in terms of a "mean free path', $\lambda$. The mean free path used is in all cases the mean free path for a nonelastic collision at the highest incident energy in the source spectrum. Since the highest incident energy in the incident source spectrum is a function of the angular interval considered (see Fig. 1), the meanfree-path values used are dependent on both the incident angular interval considered and the shield material. (The $\lambda$ values for all cases considered are given in Fig. 4.)

The omnidirectional neutron fluence per unit energy multiplied by the square of the radial distance into the shield is shown in Fig. 2 as a function of energy at a radial distance of 7 mean free paths for the case of the 0 to $30^{\circ}$ neutron spectrum incident on the various shield materials. The fluence per unit energy has been multiplied by the square of the radial distance into the shield to

\footnotetext{
† The master cross-section tape for use in the O5R Monte Carlo code, together with references to the source of the cross sections, is also available from RSIC.
} 


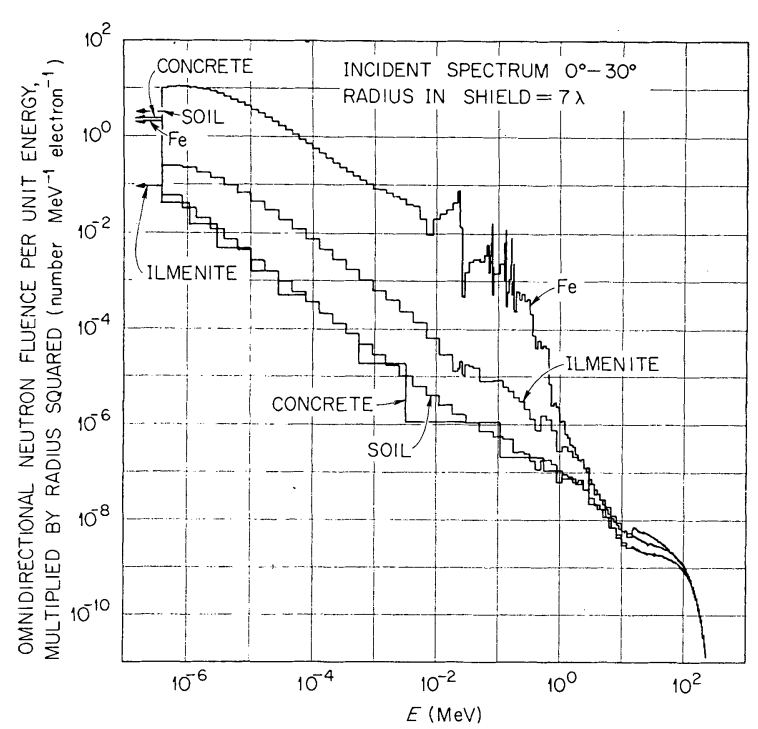

FIG. 2. Omnidirectional neutron fluence per unit energy multiplied by the square of the radial depth in the shield vs energy for various shield materials.

remove insofar as possible the geometric attenuation. The fluences per unit energy are shown at the same radial depth in mean free paths to make them insofar as possible 'physically comparable'. The actual choice of the mean free paths used, however, is somewhat arbitrary, so no very precise meaning can be given to the term 'physically comparable'. The histogram intervals shown in the figure correspond to the energy group structure used in the discrete ordinates calculations. In the case of concrete, the group structure used corresponds to that used elsewhere. ${ }^{5}$

At very high energies $(\gtrsim 100 \mathrm{MeV})$, all of the fluences per unit energy shown in Fig. 2 have the same magnitude and shape, but very substantial differences between some of the curves develop as the energy decreases. The slight increase in the fluence per unit energy in iron just above $15 \mathrm{MeV}$ is thought to be spurious. It is probably due to the fact that the differential cross-section data used for iron at energies just above $15 \mathrm{MeV}$ do not agree with the differential cross-section data used for iron just below $15 \mathrm{MeV}$. The histogram values in Fig. 2 at the lowest energies (i.e., those with arrows) represent the omnidirectional neutron fluence per unit energy multiplied by the radius squared at thermal energies, which is taken here to mean all energies $\leqq 4.14 \times 10^{-7} \mathrm{MeV}$. The concrete and soil curves have approximately the same magnitude and shape at all energies. The presence and effect of resonances in the iron cross sections are very apparent. The most striking feature shown in Fig. 2 is the very substantial difference at low energies $(\lesssim 1 \mathrm{MeV})$ between the fluence per unit energy in iron and the fluence per unit energy in the other materials. The moderating effect of the light elements in the soil and concrete shields is very apparent.

The differences in shape of the fluence per unit energy in the various materials have a very significant effect on the dose-equivalent results. The fractional contribution to the total dose equivalent from neutrons with energies less than $E$ for the spectra given in Fig. 2 is shown in Fig. 3 as a

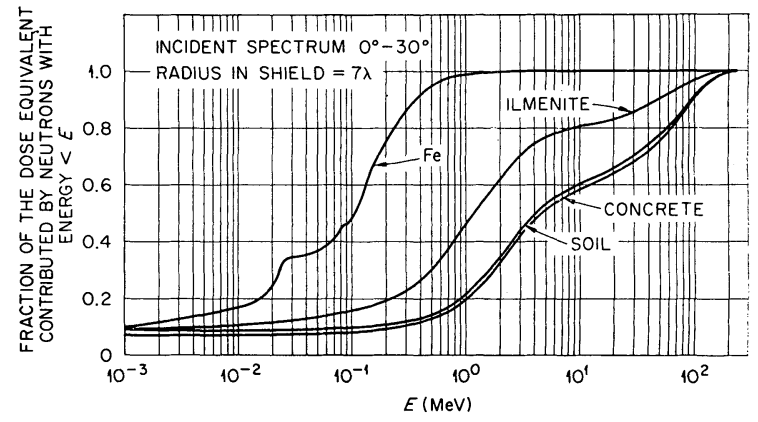

FIG. 3. Fractional contribution to the total dose equivalent from neutrons with energies less than $E$ vs $E$ for various shield materials.

function of $E$. In the case of an iron shield, almost the entire dose equivalent is contributed by neutrons with energies $\lesssim 1 \mathrm{MeV}$. In the case of soil and concrete shields on the other hand, even very high energy neutrons contribute appreciably to the dose equivalent. The results presented in Figs. 2 and 3 are for a specific radius in the shield and for a specific incident spectrum, but qualitatively similar results would be obtained at the larger depths for all of the incident spectra considered.

The dose equivalent multiplied by the radius squared is shown in Fig. 4 as a function of radius for all of the incident spectra considered and for shields of iron, ilmenite, soil, and concrete. Also shown in the figure for the 0 to $30^{\circ}$ and the 120 to $180^{\circ}$ incident spectra are the results for a shield 


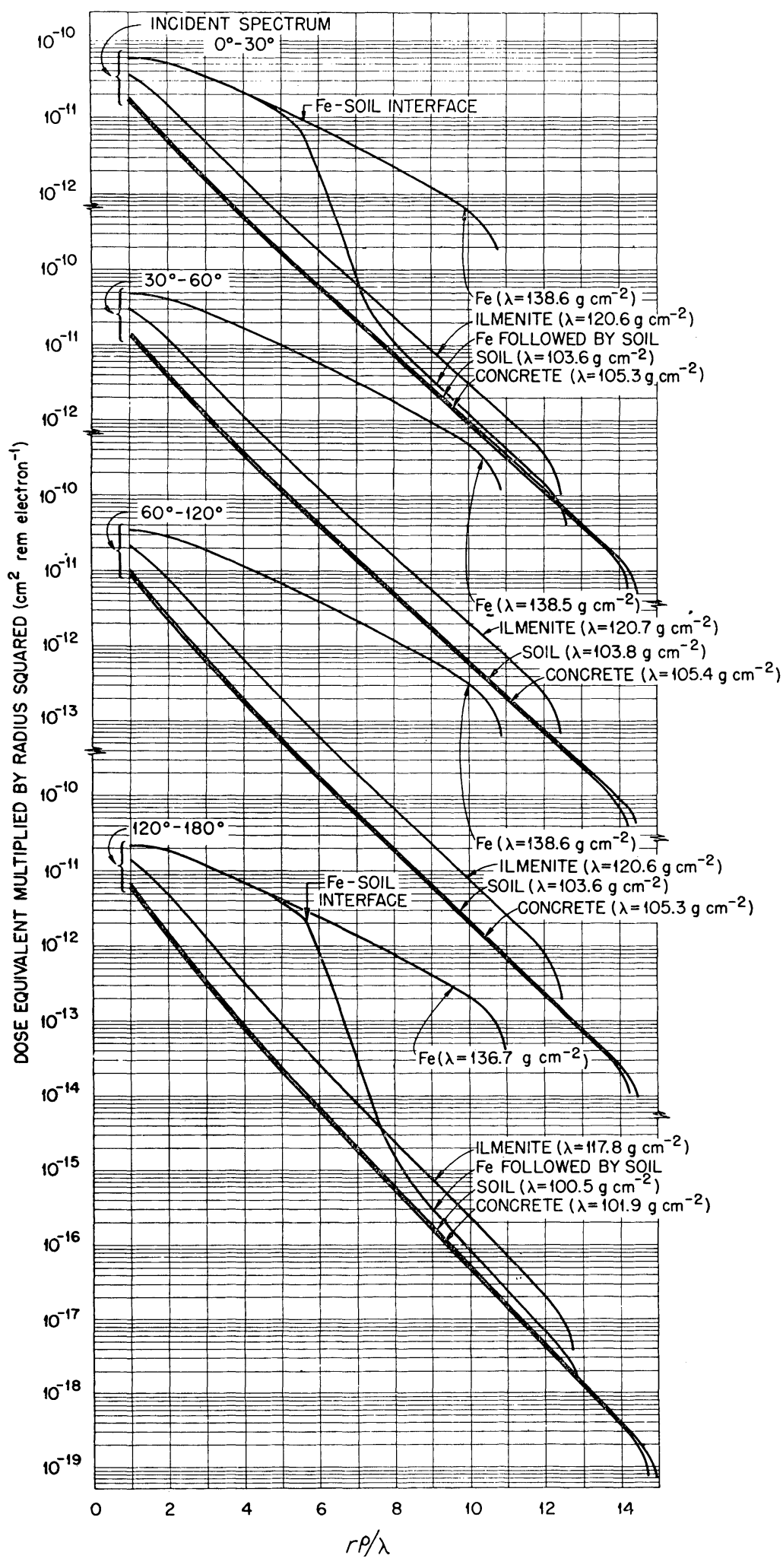

FIG. 4. Dose equivalent multiplied by the radius squared vs radius for a variety of incident spectra and shield materials. Note that the dose equivalent has been multiplied by the radius squared in $\mathrm{cm}^{2}$, but the results are plotted against the dimensionless variable $r \rho / \lambda$, where $r$ is in $\mathrm{cm}, \rho$ is in $\mathrm{g} \mathrm{cm}^{-3}$, and $\lambda$ is in $\mathrm{g} \mathrm{cm}^{-2}$. 
of $99.06 \mathrm{~cm}$ of iron followed by soil. In all cases, the shield radius was taken to be $1500 \mathrm{~g} \mathrm{~cm}^{-2}$, and the largest radius for which a value is given for each curve corresponds to $1500 \mathrm{~g} \mathrm{~cm}^{-2}$ measured in terms of the appropriate value of $\lambda$. In the case of an iron shield followed by soil, the $\lambda$ value for iron was used in obtaining abscissa values in the iron portion of the shield and the $\lambda$ value for soil was used in obtaining abscissa values in the soil portion of the shield. The abrupt decrease in the dose-equivalent curves near the end of the shield is due to the absence of an albedo fluence at the end of the shield. In all cases, the curves are shown for all radii greater than one mean free path. In considering the results, however, it must be remembered that incident neutrons $<15 \mathrm{MeV}$ were not considered and therefore the doseequivalent values given in Fig. 4 for the first few mean free paths, particularly in iron, might be underestimates for this reason.

For all of the neutron spectra considered, the curves for iron in Fig. 4 are very different from the curves for ilmenite, concrete, and soil. For a given neutron spectrum, the curves for soil and concrete are very similar. In the case of an iron shield followed by soil, the dose equivalent is the same as that for iron until near the iron-soil interface. The iron-soil curves fall below the iron curves just before the interface because the albedo fluence from soil is less than the albedo fluence from iron. In the vicinity of the interface, the dose equivalent decreases very rapidly with increasing radius and ultimately attains a magnitude and shape that are nearly the same as those for a soil shield.

\section{ACKNOWLEDGEMENTS}

Thanks are due to E. Oblow for many helpful discussions concerning the transport of low-energy neutrons in iron and to R. Q. Wright for his assistance in obtaining the crosssection data used in the calculations.

\section{REFERENCES}

1. T. A. Gabriel and R. G. Alsmiller, Jr., Nucl. Phys., B14, 303 (1969); see also T. A. Gabriel and R. G. Alsmiller, Jr., 'Photonucleon and Photopion Production from High-Energy (50 to $400 \mathrm{MeV}$ ) Electrons in Thick Copper Targets,' Report ORNL-4443, Oak Ridge National Laboratory (1969).

2. T. A. Gabriel, 'Analytic Representation of Photonucleon and Photopion Differential Yields Resulting from High-Energy Electrons $\left(50 \leqq \mathrm{E}_{0} \leqq 400 \mathrm{MeV}\right)$ Incident on an Infinite Copper Target,' Report ORNL-4442, Oak Ridge National Laboratory (1969).

3. R. G. Alsmiller, Jr., F. R. Mynatt, J. Barish, and W. W. Engle, Jr., Nucl. Sci. Eng., 36, 251 (1969).

4. R. G. Alsmiller, Jr., F. R. Mynatt, J. Barish, and W. W. Engle, Jr., Nucl. Instr. Meth., 72, 213 (1969).

5. R. W. Roussin, R. G. Alsmiller, Jr., and J. Barish, Nucl. Eng. Design, 24, 250 (1973).

6. W. W. Engle, Jr., 'A Users Manual for ANISN, a One-Dimensional Discrete Ordinates Transport Code with Anisotropic Scattering,' K-1693, Computing Technology Center, Union Carbide Corporation (1967).

7. 'RSIC Data Library Collection,' DLC-2, Radiation Shielding Information Center, Oak Ridge National Laboratory (1972).

8. E. M. Pennington and J. C. Gajniak, 'Compilation of ENDF/B Data for Magnesium, Titanium, Vanadium, Molybdenum, and Gadolinium,' Report ANL-7387, Argonne National Laboratory (1968).

9. H. W. Bertini, Nucl. Phys., 87, 138 (1966).

10. R. G. Alsmiller, Jr., M. Leimdorfer, and J. Barish, 'Analytic Representation of Nonelastic Cross Sections and Particle-Emission Spectra from Nucleon-Nucleus Collisions in the Energy Range 25 to $400 \mathrm{MeV}$, Report ORNL-4046, Oak Ridge National Laboratory (1967).

11. R. G. Alsmiller, Jr. and J. Barish, 'NCDATA-Nuclear Collision Data for Nucleon-Nucleus Collisions in the Energy Range 25 to $400 \mathrm{MeV}$,' Report ORNL-4220, Oak Ridge National Laboratory (1968).

12. H. W. Bertini, 'Monte Carlo Calculations on Intranuclear Cascades,' Report ORNL-3383, Oak Ridge National Laboratory (1963).

13. D. C. Irving et al., 'O5R, a General-Purpose Monte Carlo Neutron Transport Code,' Report ORNL-3622, Oak Ridge National Laboratory (1965).

14. International Commission on Radiation Units and Measurements, 'Radiation Protection Instrumentation and its Application,' ICRU Report 20 (1971).

Received 18 April 1973; and in final form 16 May 1973 